\title{
CYP2C19*2 Allele Carrier Status and Coronary In-stent Restenosis: Is There an Association?
}

\author{
Francesca Wirth ${ }^{1 *}$, Graziella Zahra², Robert G. Xuereb ${ }^{3}$, Christopher Barbara ${ }^{2}$, Liberato Camilleri ${ }^{4}$, \\ Albert Fenech ${ }^{3}$ and Lilian M. Azzopardi ${ }^{1}$ \\ ${ }^{1}$ Department of Pharmacy, Faculty of Medicine and Surgery, University of Malta, Msida, Malta; ${ }^{2}$ Molecular Diagnostics Unit, Depart- \\ ment of Pathology, Mater Dei Hospital, Msida, Malta; ${ }^{3}$ Cardiac Catheterisation Suite, Department of Cardiology, Mater Dei Hospital, \\ Msida, Malta; ${ }^{4}$ Department of Statistics and Operations Research, Faculty of Science, University of Malta, Msida, Malta
}

\begin{abstract}
Background and objective: The CYP2C19*2 allele is associated with reduced clopidogrel bioactivation, increasing the risk of complications after percutaneous coronary intervention $(\mathrm{PCl})$, particularly stent thrombosis. Recently published data suggests that CYP2C19*2 allele carriers have a higher risk for in-stent restenosis (ISR) after endovascular treatment. Very few studies have investigated the relationship between CYP2C19*2 and coronary ISR, with no significant association reported. The objective of this study was to assess the relationship between CYP2C19*2 allele carrier status and coronary ISR.

Methods: Patients with previous $\mathrm{PCl}$ with stenting and who were scheduled for elective $\mathrm{PCl}$ after coronary angiogram were recruited from the cardiac catheterization suite over a 12-month period. The angiography report of each patient was perused to identify patients requiring PCI due to ISR. For patients with angiography-confirmed ISR, date of previous $\mathrm{PCl}$ to the restenosed stent was noted. CYP2C19*2 genotyping was undertaken using a $\operatorname{TaqMan}^{\circ}$ Drug Metabolism assay. The association between CYP2C19*2 allele carrier status and incidence of coronary ISR within 1 year was assessed using Fisher's exact test $(p<0.05$ significance) and by calculating the odds ratio $(\mathrm{OR})$ with a $95 \%$ confidence interval $(\mathrm{CI})$.
\end{abstract}

Results: Of the 82 patients with previous $\mathrm{PCl}, 29$ (35.4\%) had angiography-confirmed ISR (12 carriers, 17 noncarriers of CYP2C19*2). In $13(44.8 \%)$ of these patients, the restenosed stent was deployed within 1 year and the patients were on clopidogrel therapy at the time of repeat $\mathrm{PCI}$ (8 carriers, 5 non-carriers of CYP2C19*2). The association between CYP2C19*2 allele carrier status and ISR within 1 year was not statistically significant (Fisher's exact $p=0.067$; OR: $4.80,95 \% \mathrm{Cl}: 0.98-23.54, p=0.053$ ).

Conclusions: Despite a higher proportion of CYP2C19*2 allele carriers exhibiting ISR within 1 year compared to non-carriers, the association was not statistically significant. This result may be attributed to the small sample size, and larger prospective studies are recommended to further assess this association.

Keywords: Clopidogrel; Coronary in-stent restenosis; $C Y P 2 C 19 * 2$ polymorphism; Percutaneous coronary intervention; Pharmacogenetic testing.

Abbreviations: BMS, bare metal stent; CI, confidence interval; CYP, cytochrome P; DES, drug-eluting stent; EM, extensive metabolizer; IM, intermediate metabolizer; ISR, in-stent restenosis; OR, odds ratio; PCI, percutaneous coronary intervention; PM, poor metabolizer.

Received: January 10, 2018; Accepted: February 23, 2018

*Correspondence to: Francesca Wirth, Department of Pharmacy, Faculty of Medicine and Surgery, University of Malta, Msida, Malta. MSD 2080. Tel: +3562340 2902: E-mail: francesca.wirth@um.edu.mt

How to cite this article: Wirth F, Zahra G, Xuereb RG, Barbara C, Camilleri L, Fenech A, Azzopardi LM. CYP2C19*2 Allele Carrier Status and Coronary In-stent Restenosis: Is There an Association? J Explor Res Pharmacol 2018;3(2):55-60. doi: 10.14218/ JERP.2018.00002

\section{Introduction}

Dual antiplatelet therapy with aspirin and a $\mathrm{P} 2 \mathrm{Y}_{12}$ receptor inhibitor is the standard treatment for patients undergoing percutaneous coronary intervention (PCI) with stent deployment to reduce the risk of post-procedural thrombotic complications. This synergistic combination therapy is typically given for 3 months after PCI for bare metal stents (BMS) and for at least 1 year with drug-eluting stents (DES). ${ }^{1-3}$ Whilst European guidelines tend to favor use of the newer $\mathrm{P}_{2} \mathrm{Y}_{12}$ receptor inhibitors prasugrel and ticagrelor as first-line agents, provided there is no high risk of bleeding ${ }^{2}$, American guidelines recommend these newer antiplatelet agents as po- 
tential alternatives to clopidogrel. ${ }^{3}$ Clopidogrel is still the most extensively used $\mathrm{P} 2 \mathrm{Y}_{12}$ receptor inhibitor after PCI, particularly due to more robust evidence, lower cost and decreased incidence of bleeding compared to the newer $\mathrm{P} 2 \mathrm{Y}_{12}$ receptor inhibitors. ${ }^{4}$

Clopidogrel is a thienopyridine inactive prodrug and requires biotransformation in the liver to form its pharmacologically active metabolite, which selectively and irreversibly antagonizes the P2 $\mathrm{Y}_{12}$ component of the adenosine diphosphate receptor on the platelet surface and consequently attenuates platelet aggregation. ${ }^{5}$ Two sequential hepatic oxidative steps are involved in clopidogrel metabolism to the active metabolite, ${ }^{5}$ with cytochrome P (CYP) $4502 \mathrm{C} 19$ being the principal enzyme involved in both steps. ${ }^{6}$ The CYP2C19 enzyme is encoded by a highly polymorphic gene and CYP2C19 single nucleotide polymorphisms have been identified as significantly and consistently associated with variability in clopidogrel response. ${ }^{6-9}$

The *2 variant allele is the most common CYP2C19 loss-offunction allele, translating to decreased CYP2C19 enzyme activity, with an estimated prevalence of $29 \%$ to $35 \%$ in Asians and $15 \%$ in Caucasians and Africans. ${ }^{7}$ Non-carriers of the $* 2$ allele (homozygous $* 1 / * 1)$ are classified as extensive metabolizers (EMs), carriers of one $* 2$ allele (heterozygous $* 1 / * 2$ ) as intermediate metabolizers (IMs) and carriers of two *2 alleles (homozygous *2/*2) as poor metabolizers (PMs) of clopidogrel. EMs efficiently metabolize clopidogrel to the active form and show the highest levels of platelet inhibition with standard $75 \mathrm{mg}$ clopidogrel dosing, while IMs and PMs show decreased inhibition of platelet aggregation. ${ }^{8}$ The $C Y P 2 C 19^{*} 2$ allele has been associated with reduced active clopidogrel metabolites and higher on-clopidogrel platelet reactivity, leading to a greater risk of adverse cardiovascular events in PMs and IMs compared to EMs. The strongest association was reported in patients with acute coronary syndrome undergoing PCI with stent implantation, where $* 2$ allele carriers had an increased risk of stent thrombosis. ${ }^{\mathbf{8}, 9}$

In-stent restenosis (ISR) is another well-documented complication of PCI. ${ }^{10}$ Although the rate of ISR decreased significantly with the use of DES compared to balloon angioplasty and BMS, it still persisted as a limiting factor for the long-term benefit of PCI, with an approximate rate of $10 \%$ in non-complex lesions. ${ }^{2,11-15}$ ISR develops through different phases involving vascular smooth muscle cell migration and proliferation, resulting in neointimal hyperplasia. ${ }^{12,15-19}$ DES-ISR typically occurs within 6 to 12 months after stent deployment, ${ }^{11}$ causing partial reocclusion of the intervention site. ${ }^{20}$ When manifested clinically as stable angina or ischemia, repeat target vessel revascularization is generally required. ${ }^{2,21,22}$ Risk factors reported to influence the extent of ISR are lesion features, including chronic total occlusion, calcification, bifurcation, small vessel diameter and long stenosis; technical factors, namely stent material, width, length and location, comorbidities such as diabetes mellitus, as well as drug resistance and/or hypersensitivity. ${ }^{12,15,20,21}$

Very few studies have investigated the relationship between CYP2C19*2 allele carrier status and ISR. Two studies published in 2014 demonstrated that the $C Y P 2 C 19^{*} 2$ variant allele is a risk factor for peripheral and vertebral artery ISR in clopidogrel-treated patients. ${ }^{23,24}$ With respect to coronary ISR, a 2015 case-match study in an Iranian patient population indicated that the prevalence of ISR during a 1-year period after PCI was higher in patients who were carriers of $C Y P 2 C 19 * 2$; however, the association did not reach statistical significance. ${ }^{25}$ In contrast, in a recent study by Ruedlinger et al. ${ }^{26}$ undertaken in Chilean patients who underwent PCI, noncarriers of the $C Y P 2 C 19 * 2$ allele had a higher incidence of ISR.

The objective of this study was to assess the relationship between $C Y P 2 C 19 * 2$ allele carrier status and coronary ISR.
Methods

\section{Ethics approval}

The study protocol was approved by the University of Malta Research Ethics Committee (19/2013).

\section{Study setting}

The study was undertaken at Mater Dei Hospital, an acute general hospital in Malta. Patients were recruited from the cardiac catheterization suite at the Department of Cardiology and genotyping was carried out at the Molecular Diagnostics Unit of the Department of Pathology.

\section{Patient recruitment, sampling and data collection}

Patients $\geq 18$ years of age, with a history of previous PCI with stent deployment and who were scheduled for elective PCI after coronary angiogram, were recruited from the cardiac catheterization suite at the time of PCI by non-probability sampling over a 12-month period, between 1 January and 31 December 2014. The coronary angiogram report of each patient was perused to identify patients requiring PCI due to angiography-confirmed ISR and those who had no ISR. For patients with angiography-confirmed ISR, the date of previous PCI to the restenosed stent was noted to establish if less than 1 year had elapsed since the previous PCI and if the patients were still on clopidogrel therapy.

After obtaining written informed consent, $5 \mathrm{~mL}$ of peripheral blood was collected from each patient in a purple-top EDTA vacutainer at the time of recruitment. A validated data collection form developed for the purpose of the study was completed for each patient to collect demographic information, cardiac risk factors, comorbidities, laboratory investigations and concomitant medications.

\section{CYP2C19*2 genotyping}

Genomic DNA was extracted using the QIAamp ${ }^{\circledR}$ DNA Mini Kit on the fully automated QIAcube (Qiagen) from $200 \mu \mathrm{L}$ of the anticoagulated whole blood sample obtained at the time of recruitment. The Taq Man ${ }^{\circledR}$ (Thermo Fisher Scientific) allelic discrimination assay (rs4244285, C_259866767_70), which involves DNA amplification and homogeneous solution hybridization using fluorescence resonance energy transfer, was used for $C Y P 2 C 19 * 2$ genotyping on the 7500 Applied Biosystems real-time PCR system. Using a 96-well PCR plate, each well had a final volume of $25 \mu \mathrm{L}$ consisting of genomic DNA, an allele-specific probe labelled with $\mathrm{VIC}^{\circledR}$ dye and another labelled with 6FAM ${ }^{\mathrm{TM}}$ dye, forward and reverse primers and TaqMan ${ }^{\circledR}$ Universal PCR Master Mix. The PCR reaction was performed as an initial denaturation at $95^{\circ} \mathrm{C}$ for $10 \mathrm{~min}$ utes, followed by 50 denaturation cycles at $92{ }^{\circ} \mathrm{C}$ for 15 seconds and annealing/extension at $60{ }^{\circ} \mathrm{C}$ for 90 seconds. Patients were genotyped as carriers or non-carriers of the CYP $2 C 19 * 2$ allele.

\section{Statistical analysis}

Data was analyzed using IBM SPSS Statistics 24. Distribution between patients who presented with coronary angiography-confirmed ISR at the time of recruitment and patients with no ISR was 


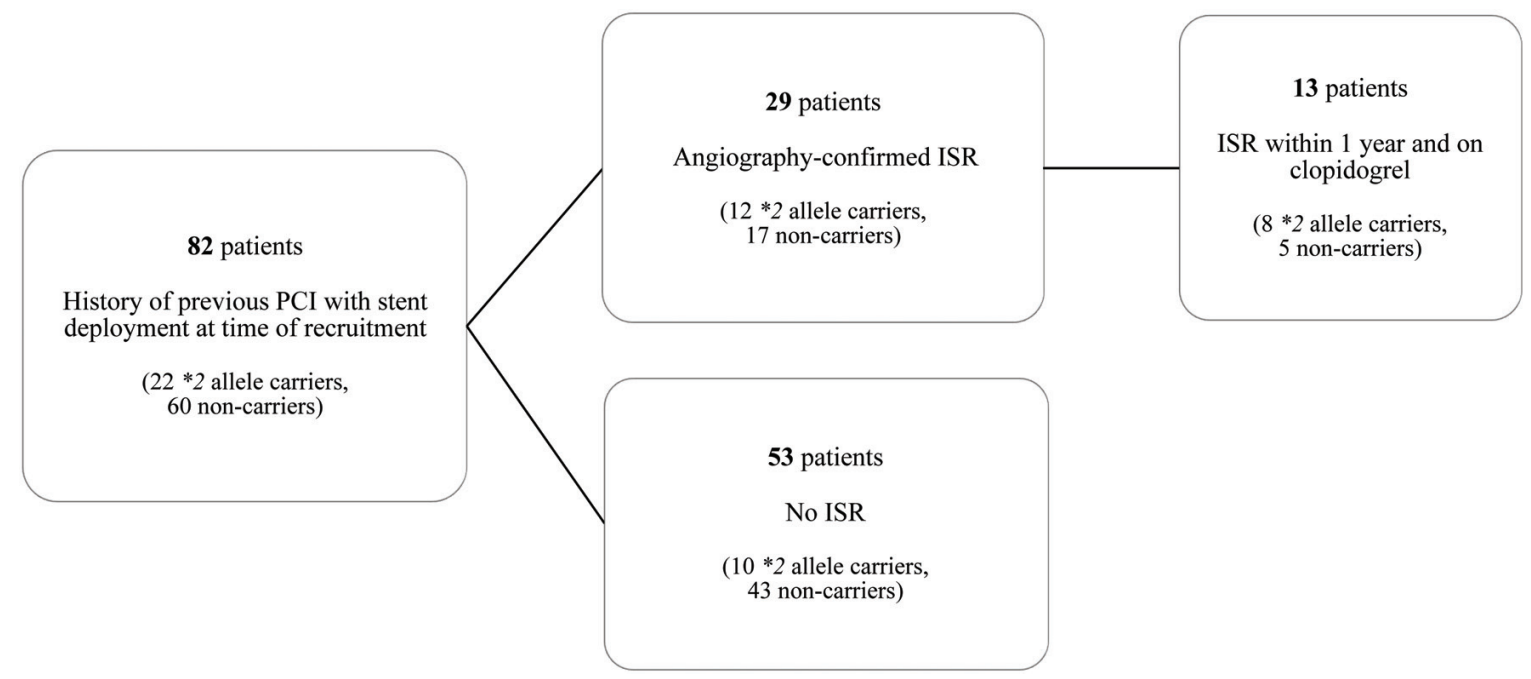

Fig. 1. In-stent restenosis at time of recruitment $(N=82)$. Abbreviations: ISR, in-stent restenosis; PCI, percutaneous coronary intervention.

tested using the one-way ANOVA test for continuous variables and the Pearson's chi-square test for categorical variables, with a $95 \%$ confidence interval (CI). Continuous variables were presented as mean \pm standard deviation, and categorical variables were presented as number of patients and percentage (\%). The association between CYP $2 C 19 * 2$ allele carrier status and incidence of coronary ISR within 1 year was assessed with cross-tabulations and the Fisher's exact test and by calculating the odds ratio (OR) with a $95 \%$ CI. A $p$-value less than 0.05 was considered statistically significant.

\section{Results}

Two hundred and fifty-two (30\%) patients out of the total 843 patients (including first PCI and history of PCI patients) who un- derwent PCI between 1 January and 31 December 2014 were recruited. Of the 252 patients, $82(32.5 \%)$ had a history of previous PCI with stent deployment and were included in the study. Out of these 82 patients, $29(35.4 \%)$ were undergoing repeat PCI due to angiography-confirmed ISR (Fig. 1). Twenty-five patients had DES-ISR, while 4 patients had BMS-ISR. The most commonly affected coronary vessels were the left anterior descending artery ( $n$ $=9)$, right coronary artery $(n=7)$ and saphenous venous graft $(n$ $=6$ ). Three patients had ISR in a bifurcation lesion. The characteristics of the patients with ISR and those with no ISR were similar $(p>0.05)$, except for $C Y P 2 C 19 * 2$ allele carrier status and renal impairment (estimated glomerular filtration rate $<60 \mathrm{~mL} / \mathrm{min} / 1.73$ $\left.\mathrm{m}^{2}\right)$, which were significantly higher in the patients with ISR ( $p$ $<0.05$ ) (Table 1). In $13(44.8 \%)$ of the 29 patients with ISR, the restenosed stent was deployed within 1 year and the patients were

Table 1. Patient characteristics $(N=82)^{\mathrm{a}}$

\begin{tabular}{llll}
\hline & ISR, $n=\mathbf{2 9}$ & No ISR, $n=\mathbf{5 3}$ & $p$-value $\mathbf{9 5 \%}$ Cl \\
\hline Age & $64.83 \pm 9.896$ & $64.45 \pm 8.915$ & 0.862 \\
Male sex & $25(86.2)$ & $40(75.5)$ & 0.250 \\
Caucasian ethnicity & $28(96.6)$ & $53(100)$ & 0.174 \\
Carrier of CYP2C19*2 allele & $12(41.4)$ & $10(18.9)$ & $0.028^{*}$ \\
eGFR $<60$, as $\mathrm{mL} / \mathrm{min} / 1.73 \mathrm{~m}^{2}$ & $12(41.4)$ & $9(17.0)$ & $0.016^{*}$ \\
Active smoking & $7(24.1)$ & $13(24.5)$ & 0.967 \\
BMI $\geq 30^{b}$, as $\mathrm{kg} / \mathrm{m}^{2}$ & $18(64.3)$ & $24(50.0)$ & 0.226 \\
Hypertension & $25(86.2)$ & $49(92.4)$ & 0.363 \\
Diabetes mellitus & $19(65.5)$ & $30(56.6)$ & 0.429 \\
Dyslipidemia & $26(89.7)$ & $50(94.3)$ & 0.435 \\
$\geq 2$ previous PCl & $12(41.4)$ & $19(35.8)$ & 0.624 \\
$>1$ vessel stented & $10(34.5)$ & $13(24.5)$ & 0.337 \\
Previous bypass graft surgery & $11(37.9)$ & $12(22.6)$ & 0.142 \\
\hline
\end{tabular}

avalues are expressed as number of patients (\%), except age which is expressed as mean \pm standard deviation; ${ }^{b}(\%)$ is out of a total of 28 patients for ISR and out of 48 patients for no ISR. *Reaching statistical significance $(p<0.05)$. Abbreviations: $\mathrm{Cl}$, confidence interval; eGFR, estimated glomerular filtration rate; ISR, in-stent restenosis; PCI, percutaneous coronary intervention. 
Table 2. CYP2C19*2 allele carrier status versus ISR within 1 year: Cross-tabulation

\begin{tabular}{|c|c|c|c|c|c|}
\hline & & & \multicolumn{2}{|c|}{ ISR within 1 year } & \multirow{2}{*}{ Total } \\
\hline & & & Yes & No & \\
\hline \multirow{5}{*}{ Carrier of $\mathrm{CYP} 2 \mathrm{C} 19 * 2$ allele } & \multirow{2}{*}{ Yes } & Number of patients & 8 & 4 & 12 \\
\hline & & Percentage & 66.7 & 33.3 & 100.0 \\
\hline & \multirow{3}{*}{ No } & Number of patients & 5 & 12 & 17 \\
\hline & & Percentage & 29.4 & 70.6 & 100.0 \\
\hline & & Total & 13 & 16 & 29 \\
\hline \multicolumn{6}{|c|}{$p=0.067$ (Fisher's exact 2 -sided). Odds ratio: $4.80,95 \%$ confidence interval: $0.98-23.54, p=0.053$. } \\
\hline
\end{tabular}

Abbreviation: ISR, in-stent restenosis.

on clopidogrel therapy at the time of repeat PCI (Fig. 1).

Of the 29 patients with ISR, 12 were carriers and 17 were noncarriers of the CYP $2 C 19 * 2$ allele. Eight $(66.7 \%)$ of the $12 * 2$ allele carriers had ISR within 1 year and were on clopidogrel therapy at the time of repeat PCI. Only 5 (29.4\%) out of the 17 non-carriers presented with ISR within 1 year. Although the difference between the two proportions $(37.3 \%)$ is large, the association between $C Y P 2 C 19 * 2$ allele carrier status and ISR within 1 year was not statistically significant (Fisher's exact $p=0.067$; OR: 4.80, 95\% CI: 0.98-23.54, $p$ $=0.053)($ Table 2$)$.

\section{Discussion}

The findings from this study show that despite a higher proportion of $C Y P 2 C 19 * 2$ allele carriers exhibiting coronary ISR within 1 year compared to non-carriers, and $* 2$ allele carriers being 4.08-times more likely to exhibit ISR than non-carriers, the association between $C Y P 2 C 19 * 2$ allele carrier status and development of coronary ISR was not statistically significant $(p>0.05)$. Our findings are similar to Nozari et $a l .{ }^{25}$ who reported that the prevalence of ISR after PCI was higher in $* 2$ allele carriers but without a statistically significant relationship, and contrast to Ruedlinger et al., ${ }^{26}$ who identified non-carriers of the $* 2$ allele with higher incidence of coronary ISR.

According to Mitra and Agrawal, ${ }^{12}$ the overall pathogenesis of ISR is not entirely understood and "prevention of ISR appears to be a multipronged attack as no therapeutic magic bullet exists to block all the processes in one go". In clinical practice, the importance of ISR is well-known. ${ }^{10}$ Reducing the incidence of ISR is crucial, since ISR limits the long-term benefit of PCI by affecting the quality of life of the patient due to physical limitations caused by angina, ${ }^{26}$ and augments health care costs due to the need for repeat revascularisation. ${ }^{27,28}$

Stent implantation causes injury to the arterial vessel wall, stimulating platelet activation through a number of pathways, ${ }^{17,29,30}$ hence the rationale for prescribing antiplatelet therapy after PCI. Platelets are reported to be involved in the pathophysiology of ISR, ${ }^{12,18,29,30}$ so the higher proportion of $C Y P 2 C 19 * 2$ allele carriers exhibiting coronary ISR within 1 year compared to non-carriers may be explained in terms of inadequate platelet inhibition after PCI when these decreased clopidogrel responders are treated with clopidogrel. Recommendations for CYP2C19 genotype and clopidogrel therapy state that a more potent $\mathrm{P} 2 \mathrm{Y}_{12}$ receptor inhibitor with no or little interindividual response variability, such as prasugrel or ticagrelor, unless contraindicated, rather than clopidogrel, should be prescribed in carriers of the CYP 2 C19*2 allele, while non-carriers should be prescribed clopidogrel at the standard $75 \mathrm{mg}$ dose. ${ }^{8,31}$
The $p$-value is highly dependent on the sample size and it is very unlikely to obtain statistical significance when the sample size is small, unless the difference between the proportions is considerable. The Fisher's exact $p$-value obtained in our study (0.067) exceeds the 0.05 level of significance by only a small margin, despite the very small sample size (29 patients with ISR). Compared to our study, Nozari et al. ${ }^{25}$ had a larger sample of patients with ISR (50 patients) and a higher $p$-value $(0.273)$ was obtained. Hence, given the small sample size, the findings from our study should not be ignored. Since the $p$-value obtained in our study exceeds the 0.05 level of significance by only a small margin, it is likely that a slightly larger sample size would have yielded a significant association between the two categorical variables.

The restricted sample size in this study limited power of the data and the ability to draw definite conclusions. Larger prospective case-control studies are recommended to further assess this reported association, which may open up new possible explanations of the pathophysiology of ISR and substantiate the role of CYP 2 C19*2 genotyping with respect to antiplatelet therapy in patients undergoing PCI. The possibility that measured and unmeasured clinical and angiographic factors that may predispose to ISR development, such as lesion features, technical details of the PCI and comorbidities, which may have contributed to this association cannot be excluded. One measured predisposing factor is renal impairment, which was significantly higher in patients with ISR. This association is supported by other studies that have linked renal insufficiency with increased incidence of coronary ISR. ${ }^{32-36}$ On the other hand, in a study by Best et al., ${ }^{37}$ ISR was not increased with chronic kidney disease.

\section{Future research prospective}

ISR is a limiting factor for the long-term benefit of PCI. When manifested clinically, repeat target vessel revascularization is generally required. The higher proportion of $C Y P 2 C 19 * 2$ allele carriers exhibiting ISR within 1 year compared to non-carriers is an interesting finding regarding the pharmacogenetic implications of clopidogrel and the incidence of coronary ISR. Larger prospective studies are recommended to assess this finding further, which may open up new possible explanations of the pathophysiology of ISR and support the utilization of CYP $2 C 19 * 2$ genotyping for the personalization of antiplatelet therapy in patients undergoing PCI.

\section{Conclusions}

The higher proportion of $C Y P 2 C 19 * 2$ allele carriers exhibiting 
ISR within 1 year compared to non-carriers is an interesting finding regarding the association of clopidogrel metabolism and the prevalence of coronary ISR. Since no significant association was found, which may be attributed to the small sample size, larger prospective studies are recommended to further assess this association.

\section{Acknowledgments}

This research was financially supported by the University of Malta's Faculty of Medicine and Surgery Dean's Initiative, Scientech Ltd., E.J. Busuttil Ltd., and the Malta Heart Foundation. This research was carried out in collaboration with all the consultant cardiologists and staff at the Department of Cardiology at Mater Dei Hospital. The authors would like to thank the Pharmacogenetics Laboratory of the Institute of Biochemistry in the Faculty of Medicine at the University of Ljubljana, Slovenia, and the Department of Clinical Pharmacy and Toxicology at the Leiden University Medical Center, The Netherlands, for providing the positive controls.

\section{Conflict of interest}

The authors have no conflict of interest related to this publication.

\section{Author contributions}

Study design (FW, AF, LMA), clinical support (RGX, AF), performance of experiments and laboratory support (FW, GZ, CB), data analysis (FW, LC, LMA), manuscript writing (FW). All authors reviewed and approved the manuscript.

\section{References}

[1] Pant S, Neupane P, Ramesh KC, Barakoti M. Post-percutaneous coronary intervention antiplatelet therapy: Current perceptions, prospects and perplexity. Cardiol J 2011;18(6):712-717.

[2] Windecker S, Kolh P, Alfonso F, Collet JP, Cremer J, Falk V, et al. 2014 ESC/EACTS Guidelines on myocardial revascularization: The Task Force on Myocardial Revascularization of the European Society of Cardiology (ESC) and the European Association for Cardio-Thoracic Surgery (EACTS) developed with the special contribution of the European Association of Percutaneous Cardiovascular Interventions (EAPCI). Eur Heart J 2014;35(37):2541-2619. doi:10.1093/eurheartj/ ehu278.

[3] Amsterdam EA, Wenger NK, Brindis RG, Casey DE Jr, Ganiats TG, Holmes DR Jr, et al. 2014 ACC/AHA guideline for the management of patients with non-ST-elevation acute coronary syndromes: A report of the American College of Cardiology/American Heart Association Task Force on Practice Guidelines. J Am Coll Cardiol 2014;64(24):e139e228. doi:10.1016/j.jacc.2014.09.017.

[4] Garabedian T, Alam S. High residual platelet reactivity on clopidogrel: Its significance and therapeutic challenges overcoming clopidogrel resistance. Cardiovasc Diagn Ther 2013;3(1):23-37. doi:10.3978/j. issn.2223-3652.2013.02.06

[5] Sangkuhl K, Klein TE, Altman RB. Clopidogrel pathway. Pharmacogenet Genomics 2010;20(7):463-465. doi:10.1097/ FPC.0b013e3283385420.

[6] Kazui M, Nishiya Y, Ishizuka T, Hagihara K, Farid NA, Okazaki O, et al. Identification of the human cytochrome P450 enzymes involved in the two oxidative steps in the bioactivation of clopidogrel to its phar- macologically active metabolite. Drug Metab Dispos 2010;38(1):9299. doi:10.1124/dmd.109.029132.

[7] Scott SA, Sangkuhl K, Shuldiner AR, Hulot JS, Thorn CF, Altman RB, et al. PharmGKB summary: Very important pharmacogene information for cytochrome P450, family 2, subfamily C, polypeptide 19. Pharmacogenet Genomics 2012;22(2):159-165. doi:10.1097/ FPC.0b013e32834d4962.

[8] Scott SA, Sangkuhl K, Stein CM, Hulot JS, Mega JL, Roden DM, et al. Clinical Pharmacogenetics Implementation Consortium guidelines for CYP2C19 genotype and clopidogrel therapy: 2013 update. Clin Pharmacol Ther 2013;94(3):317-323. doi:10.1038/clpt.2013.105.

[9] Yang Y, Lewis JP, Hulot JS, Scott SA. The pharmacogenetic control of antiplatelet response: Candidate genes and CYP2C19. Expert Opin Drug Metab Toxicol 2015;11(10):1599-1617. doi:10.1517/17425255 .2015 .1068757

[10] Wilson JM. Unintended consequences: Avoiding restenosis and stent thrombosis. Tex Heart Inst J 2010;37(3):341-342.

[11] Htay T, Liu MW. Drug-eluting stent: A review and update. Vasc Health Risk Manag 2005;1(4):263-276.

[12] Mitra AK, Agrawal DK. In stent restenosis: Bane of the stent era. J Clin Pathol 2006;59(3):232-239. doi:10.1136/jcp.2005.025742.

[13] Dibra A, Kastrati A, Alfonso F, Seyfarth M, Pérez-Vizcayno MJ, Mehill $\mathrm{J}$, et al. Effectiveness of drug-eluting stents in patients with bare-metal in-stent restenosis: Meta-analysis of randomized trials. J Am Coll Cardiol 2007;49(5):616-623. doi:10.1016/j.jacc.2006.10.049.

[14] Garg S, Serruys PW. Coronary stents: Current status. J Am Coll Cardiol 2010;56(10):S1-S42. doi:10.1016/j.jacc.2010.06.007.

[15] Farooq V, Gogas BD, Serruys PW. Restenosis: Delineating the numerous causes of drug-eluting stent restenosis. Circ Cardiovasc Interv 2011;4(2):195-205. doi:10.1161/CIRCINTERVENTIONS.110.959882.

[16] Lowe HC, Oesterle SN, Khachigian LM. Coronary in-stent restenosis: Current status and future strategies. J Am Coll Cardiol 2002;39(2):183-193. doi:10.1016/S0735-1097(01)01742-9.

[17] Schwartz RS, Henry TD. Pathophysiology of coronary artery restenosis. Rev Cardiovasc Med 2002;3(5):S4-S9.

[18] Shah PK. Inflammation, neointimal hyperplasia, and restenosis: As the leukocytes roll, the arteries thicken. Circulation 2003;107(17):21752177. doi:10.1161/01.CIR.0000069943.41206.BD.

[19] Kibos A, Campeanu A, Tintoiu I. Pathophysiology of coronary artery in-stent restenosis. Acute Card Care 2007;9(2):111-119. doi:10.1080/17482940701263285.

[20] Dangas GD, Claessen BE, Caixeta A, Sanidas EA, Mintz GS, Mehran R. In-stent restenosis in the drug-eluting stent era. J Am Coll Cardiol 2010;56(23):1897-1907. doi:10.1016/j.jacc.2010.07.028.

[21] Dangas G, Kuepper F. Restenosis: Repeat narrowing of a coronary artery: Prevention and treatment. Circulation 2002;105(22):25862587. doi:10.1161/01.CIR.0000019122.00032.DF.

[22] Lee MS, Pessegueiro A, Zimmer R, Jurewitz D, Tobis J. Clinical presentation of patients with in-stent restenosis in the drug-eluting stent era. J Invasive Cardiol 2008;20(8):401-403.

[23] Guo B, Tan Q, Guo D, Shi Z, Zhang C, Guo W. Patients carrying CYP $2 \mathrm{C} 19$ loss of function alleles have a reduced response to clopidogrel therapy and a greater risk of in-stent restenosis after endovascular treatment of lower extremity peripheral arterial disease. J Vasc Surg 2014;60(4):993-1001. doi:10.1016/j.jvs.2014.03.293.

[24] Lin YJ, Li JW, Zhang MJ, Qian L, Yang WJ, Zhang CL, et al. The association between CYP 2C19 genotype and of in-stent restenosis among patients with vertebral artery stent treatment. CNS Neurosci Ther 2014;20(2):125-130. doi:10.1111/cns.12173.

[25] Nozari $Y$, Vosooghi S, Boroumand M, Poorhosseini $H$, Nematipour $\mathrm{E}$, Salarifar M, et al. The impact of cytochrome P450 2 C19 polymorphism on the occurrence of one-year in-stent restenosis in patients who underwent percutaneous coronary intervention: A casematch study. Anatol J Cardiol 2015;15(5):348-353. doi:10.5152/ akd.2014.5418.

[26] Ruedlinger J, Prado Y, Zambrano T, Saavedra N, Bobadilla B, Potthoff $\mathrm{M}$, et al. CYP2C19*2 polymorphism in Chilean patients with in-stent restenosis development and controls. Biomed Res Int 2017;2017:5783719. doi:10.1155/2017/5783719.

[27] Ryan J, Cohen DJ. Are drug-eluting stents cost-effective? It depends on whom you ask. Circulation 2006;114(16):1736-1743. 
J Explor Res Pharmacol

doi:10.1161/CIRCULATIONAHA.105.546010.

[28] Clark MA, Bakhai A, Pelletier EM, Cohen DJ. Clinical and economic effects of coronary restenosis after percutaneous coronary intervention in a managed care population. Manag Care 2005;14(4):42-51.

[29] Chandrasekar B, Tanguay JF. Platelets and restenosis. J Am Coll Cardiol 2000;35(3):555-562. doi:10.1016/S0735-1097(99)00596-3.

[30] Sangkuhl K, Shuldiner AR, Klein TE, Altman RB. Platelet aggregation pathway. Pharmacogenet Genomics 2011;21(8):516-521. doi:10.1097/FPC.0b013e3283406323.

[31] Swen JJ, Nijenhuis M, de Boer A, Grandia L, Maitland-van der Zee $\mathrm{AH}$, Mulder $\mathrm{H}$, et al. Pharmacogenetics: from bench to byte: An update of the guidelines. Clin Pharmacol Ther 2011;89(5):662-673. doi:10.1038/clpt.2011.34.

[32] Sadeghi HM, Stone GW, Grines CL, Mehran R, Dixon SR, Lansky AJ, et al. Impact of renal insufficiency in patients undergoing primary angioplasty for acute myocardial infarction. Circulation 2003;10(22):27692775. doi:10.1161/01.CIR.0000103623.63687.21.

[33] Attallah N, Yassine L, Fisher K, Yee J. Risk of bleeding and restenosis
Wirth F. et al: CYP2C19*2 and coronary in-stent restenosis

among chronic kidney disease patients undergoing percutaneous coronary intervention. Clin Nephrol 2005;64(6):412-418.

[34] El-Menyar AA, Al Suwaidi J, Holmes DR Jr. Use of drug-eluting stents in patients with coronary artery disease and renal insufficiency. Mayo Clin Proc 2010;85(2):165-171. doi:10.4065/mcp.2009.0314.

[35] Sukhija R, Aronow WS, Palaniswamy C, Singh T, Sukhija R, Kalapatapu $\mathrm{K}$, et al. Major adverse cardiac events in patients with moderate to severe renal insufficiency treated with first-generation drugeluting stents. Am J Cardiol 2010;105(3):293-296. doi:10.1016/j. amjcard.2009.09.031.

[36] Aoyama Y, Hirayama H, Ishii H, Kobayashi K, Ishikawa K, Takigawa M, et al. Impact of chronic kidney disease on a re-percutaneous coronary intervention for sirolimus-eluting stent restenosis. Coron Artery Dis 2012;23(8):528-532. doi:10.1097/MCA.0b013e3283599463.

[37] Best PJ, Berger PB, Davis BR, Grines CL, Sadeghi HM, Williams BA, et al. Impact of mild or moderate chronic kidney disease on the frequency of restenosis: Results from the PRESTO trial. J Am Coll Cardiol 2004;44(9):1786-1791. doi:10.1016/j.jacc.2004.07.052. 